\title{
Study of equation of state and fluctuations at non zero magnetic field in the hadron resonance gas model
}

\author{
Abhijit Bhattacharyya ${ }^{a}$ Sanjay K. Ghosh ${ }^{b}$ Rajarshi Ray $^{b}$ and Subhasis Samanta ${ }^{* b}$ \\ ${ }^{a}$ Department of Physics, University of Calcutta \\ 92, A. P. C. Road, Kolkata - 700009, India \\ ${ }^{b}$ Center for Astroparticle Physics \& Space Science, Bose Institute \\ Block-EN, Sector-V, Salt Lake, Kolkata-700091, India \\ \& \\ Department of Physics, Bose Institute \\ 93/1, A. P. C Road, Kolkata - 700009, India \\ E-mail: abhattacharvvacuegmail.com, sanjavejcbose.ac.in \\ rajarshidjcbose.ac.in subhasis.samantegmail.com
}

\begin{abstract}
We present temperature dependence of various thermodynamic observables including the pressure, energy density, entropy density, specific heat and fluctuations of different conserved charges using Hadron Resonance Gas (HRG) model at non-zero magnetic field. We observe that all the thermodynamic quantities as well as fluctuations get modified in presence of magnetic field.
\end{abstract}

7th International Conference on Physics and Astrophysics of Quark Gluon Plasma

1-5 February, 2015

Kolkata, India

\footnotetext{
* Speaker.
} 


\section{Introduction}

Heavy Ion collisions (HIC) are investigated both theoretically and experimentally to understand the properties of nuclear matter at extreme conditions. One of the most important issues addressed in HIC is the possibility for nuclear matter to undergo a phase transitions to quark matter. At low baryon density and high temperature nuclear matter is expected to smoothly cross over to a quark gluon plasma (QGP) phase. Whereas, at high baryon density and low temperature the system is expected to have a first order phase transition. The study of the effect of magnetic field on the phase transition in neutron stars has been a topic of interest, both theoretically as well as observationally, for a long time. In fact, several previous works have shown that the large magnetic

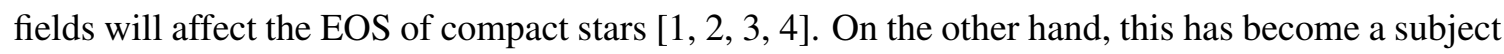
of intense research also in HIC experiments for last few years because of the presence of strong magnetic fields created by spectator particles in non-central heavy-ion collisions. The estimated magnetic field may reach up to order of $0.1 m_{\pi}^{2}, m_{\pi}^{2}, 15 m_{\pi}^{2}$ for SPS, RHIC and LHC energies respectively [[]]. Such high magnetic fields may exist only in the interior of the neutron stars and might have been present in the early universe.

Magnetic fields can induce many interesting phenomena in QCD matter, such as chiral magnetic effect (CME). The CME corresponds to the phenomenon of electric charge separation, induced by chirality imbalance, along an external magnetic field. This chiral imbalance originates due to the interaction between quarks and topologically non-trivial gluonic fields, instantons and sphalerons which also results in $\mathrm{P}$ and $\mathrm{CP}$ violation [ $\mathrm{G}, \mathrm{\square}, \mathbf{8}]$. Effects of charge separation has been studied in lattice studies [Q]. Such a charge separation has also been measured experimentally

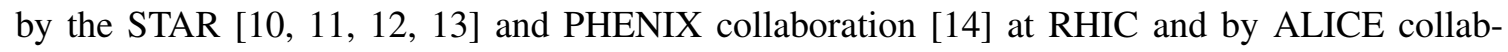
oration [15] at LHC. On the other hand, magnetic catalysis [ए6] and inverse magnetic catalysis [ㄱ] ㅁ] can affect the phase diagram of QCD matter. The modification of QCD phase diagram in presence of magnetic field has been extensively studied using lattice QCD (LQCD) [एQC, [0]] and model calculations [2], 22, 23], 24, 25]. In this work we plan to study the equation of state (EOS) and fluctuations of strongly interacting matter using the Hadron Resonance Gas (HRG)

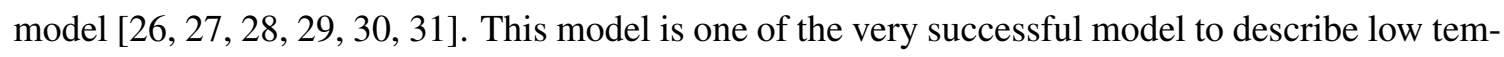
perature region of the EOS. HRG model has also been successful in describing the particle yields produced in heavy ion collision experiments from AGS to RHIC energies.

\section{HRG model in presence of magnetic field}

The grand canonical partition function of a non-interacting hadron resonance gas can be written as sum of partition functions $\ln Z_{i}^{i d}$ of all hadrons and resonances up to mass $3 \mathrm{GeV}$

$$
\ln Z^{i d}=\sum_{i} \ln Z_{i}^{i d}
$$

where $i d$ refers to ideal i.e., non-interacting HRG. For $i$ th particle,

$$
\ln Z_{i}^{i d}=\frac{V g_{i}}{(2 \pi)^{3}} \int \pm d^{3} p \ln \left[1 \pm \exp \left(-\left(E_{i}-\mu_{i}\right) / T\right)\right],
$$


where $V$ is the volume of the system, $g_{i}$ is the degeneracy factor, $T$ is the temperature, $E_{i}=$ $\sqrt{p^{2}+m_{i}^{2}}$ is the single particle energy, $m_{i}$ is the mass and $\mu_{i}=B_{i} \mu_{B}+S_{i} \mu_{S}+Q_{i} \mu_{Q}$ is the chemical potential. In the last expression, $B_{i}, S_{i}, Q_{i}$ are respectively the baryon number, strangeness and charge of the particle, $\mu$ 's are corresponding chemical potentials. The upper and lower signs corresponds to baryons and mesons respectively. The partition function is the basic quantity from which one can calculate various thermodynamic quantities of the thermal system. The pressure $P_{i}$ (in the thermodynamic limit, $V \rightarrow \infty$ ), energy density $\varepsilon_{i}$, entropy density $s_{i}$ and specific heat $C_{v}$ can be calculated as,

$$
P_{i}=\frac{T}{V} \ln Z_{i}, \quad \varepsilon_{i}=-\frac{1}{V}\left(\frac{\partial \ln Z_{i}}{\partial \frac{1}{T}}\right)_{\frac{\mu}{T}}, s_{i}=\frac{1}{V}\left(\frac{\partial\left(T \ln Z_{i}\right)}{\partial T}\right)_{V, \mu}, C_{v_{i}}=\frac{\partial \varepsilon_{i}}{\partial T} .
$$

The $n^{\text {th }}$ order susceptibility is defined as $\chi_{q}^{n}=\frac{1}{V T^{3}} \frac{\partial^{n}\left(\ln Z_{q}^{i d}\right)}{\partial\left(\frac{\mu_{q}}{T}\right)^{n}}$, where $\mu_{q}$ is the chemical potential for conserved charge $q$. It is well known that in the presence of a constant magnetic field along a particular axis, say along the $\mathrm{z}$ axis, the path of the charged particle will be a helix whose axis lies along the $\mathrm{z}$ axis and whose projection on the $\mathrm{x}-\mathrm{y}$ plane is a circle. The circular motion in $\mathrm{x}-\mathrm{y}$ plane is due to the Lorentz force experienced by the particle. For a constant strong magnetic field $\left(B_{z}\right)$ along z-axis, the single particle energy eigen value is given by [B]]

$$
E_{i}^{B_{z}}=\sqrt{p_{z}^{2}+m_{i}^{2}+\left|q_{i}\right| B_{z}\left(2 n+2 s_{z}+1\right)}
$$

where $p_{z}$ is the component of particle momentum along the direction of the external magnetic field, $q_{i}=Q_{i} e$ is the charge of the particle and $e$ is the charge of the electron, $n$ is a integer which runs from zero to infinity in step of unity for allowed Landau levels, $s_{z}$ are the components of spin $s$ in the direction of magnetic field. For a given $s$, there are $2 s+1$ possible values of $s_{z}$. The gyromagnetic ratios are taken as $g_{h}=2\left|q_{h} / e\right|$ for all charged hadrons [25]. Therefore, the partition function for $i$ th particle in presence of magnetic field can be written as [B3]],

$$
\begin{aligned}
\ln Z_{i} & =\frac{V g_{i}}{(2 \pi)^{3}} \int \pm d^{3} p \ln \left[1 \pm \exp \left(-\left(E_{i}-\mu_{i}\right) / T\right)\right], \quad \text { for } Q_{i}=0 \\
& =\frac{V g_{i}^{\prime}}{2 \pi^{2}}\left|Q_{i}\right| e B_{z} \sum_{n} \sum_{s_{z}} \int_{0}^{\infty} \pm d p_{z} \ln \left[1 \pm \exp \left(-\left(E_{i}^{B_{z}}-\mu_{i}\right) / T\right)\right], \quad \text { for } Q_{i} \neq 0
\end{aligned}
$$

where $g_{i}^{\prime}$ is the degeneracy other than spin. So partition functions of charged particles will be modified by the magnetic field whereas that of neutral particles will remain same as that of ideal HRG.

\section{Results and discussions}

In Fig. $\square$ we have shown variation of pressure $P$, energy density $\varepsilon$, entropy density $s$ and specific heat $C_{V}$ with temperature in presence of magnetic field. We have chosen three values of the magnetic fields namely : $e B_{z}=0.1,0.2$ and $0.25 \mathrm{GeV}^{2}$. It can be seen from Fig. $\square$ that $P, \varepsilon, s$ and $C_{V}$ increase with increase in magnetic field.

Figure $\square$ shows variation of second $\operatorname{order}\left(\chi^{2}\right)$ and fourth order $\left(\chi^{4}\right)$ susceptibilities of different conserved charges. At low $T$, dominating contribution to $\chi_{B}$ comes from protons and neutrons. 

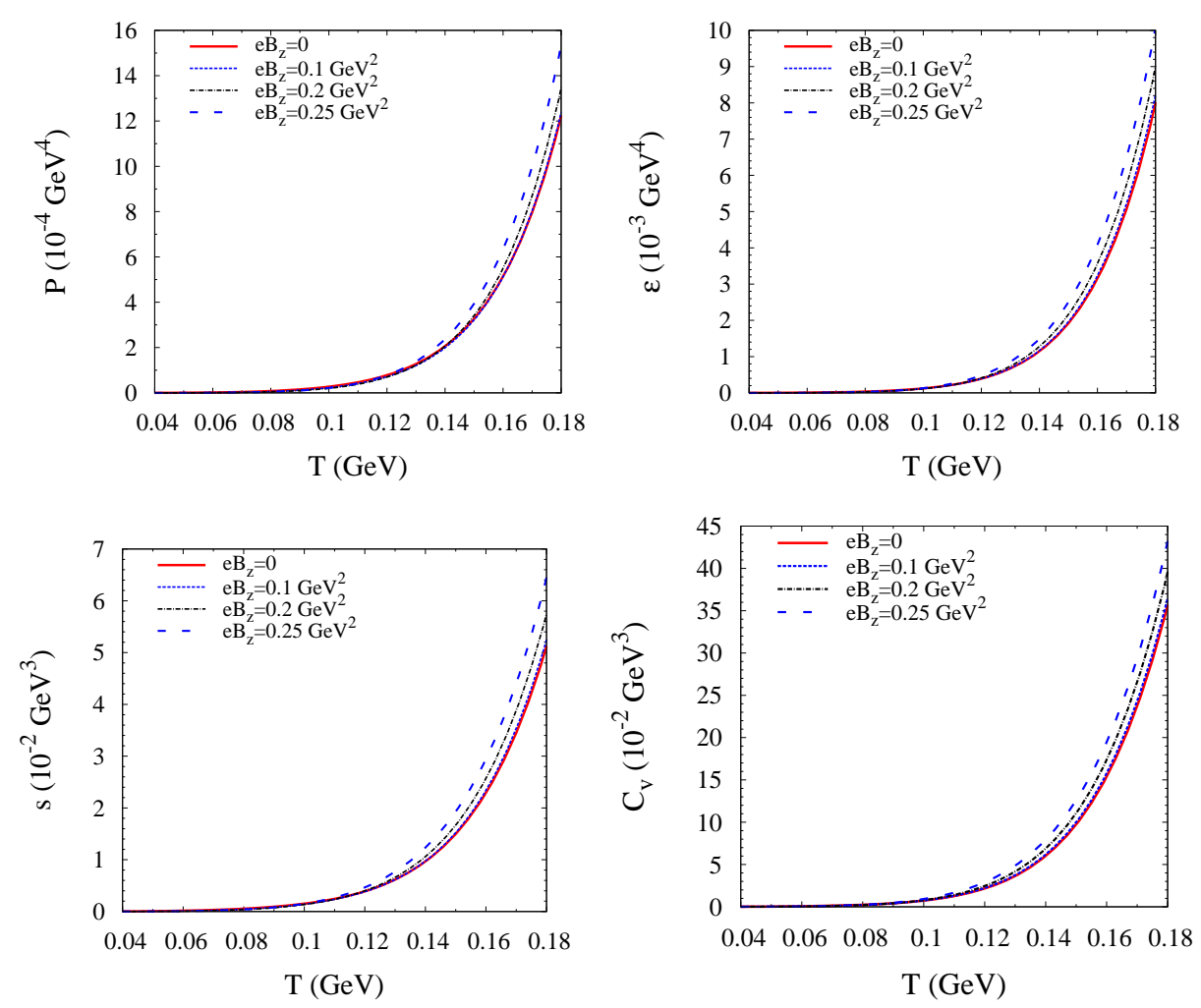

Figure 1: (Color online) Thermodynamic quantities as a function of temperature at $\mu=0$.

With increase in $T$, other heavier baryons populate and contribute to $\chi_{B}$ and hence susceptibilities increase with increase in temperature. Further, the population of charged baryons increase with the magnetic field. As a result the susceptibilities increase with the increase in magnetic field. In the middle row of Fig. $\square$ we have plotted the variation of second order $\left(\chi_{S}^{2}\right)$ and fourth order $\left(\chi_{S}^{4}\right)$ susceptibilities of strange quantum number with $T$ at $\mu=0$. The dominant contribution to $\chi_{S}^{2}$ and $\chi_{S}^{4}$ at low temperatures come from kaons. Since $K^{ \pm}$are spin zero particles, their populations get suppressed in presence of $B$. With increase in $T$, other strange mesons, like $K^{*}$ (spin one), starts populating the system. In presence of magnetic field populations of $K^{* \pm}$ increases. As the contributions $K^{ \pm}$and $K^{* \pm}$ to the susceptibilities are opposite in nature, in presence of $B$, up to certain temperature $(T<0.12 \mathrm{GeV})$, susceptibilities for strange quantum number do not get affected much by the magnetic field. At higher temperatures, other strange hadrons (like $\Lambda, \Sigma$ ) start populating the system. However, contribution from $\Lambda$ is unaffected by magnetic field as it is an uncharged particle. On the other hand population of $\Sigma^{ \pm}$increases with increase of magnetic field. Furthermore, at high temperature and high magnetic field, the contribution of $K^{* \pm}$, to the susceptibilities, is much more compared to that for $K^{ \pm}$. This also makes the susceptibilities increase with magnetic field. The bottom row of Fig. $\square$ shows variation of second order $\left(\chi_{Q}^{2}\right)$ and fourth order $\left(\chi_{Q}^{4}\right)$ susceptibilities for net electric charge with $T$ at $\mu=0$. The dominant contribution to the susceptibilities of conserved electric charges at low temperatures come from pions (lightest hadron) which are spin zero particles. Next dominating contributions are coming from kaons which are also spin zero particles. Therefore, the populations of these particles get suppressed in presence 

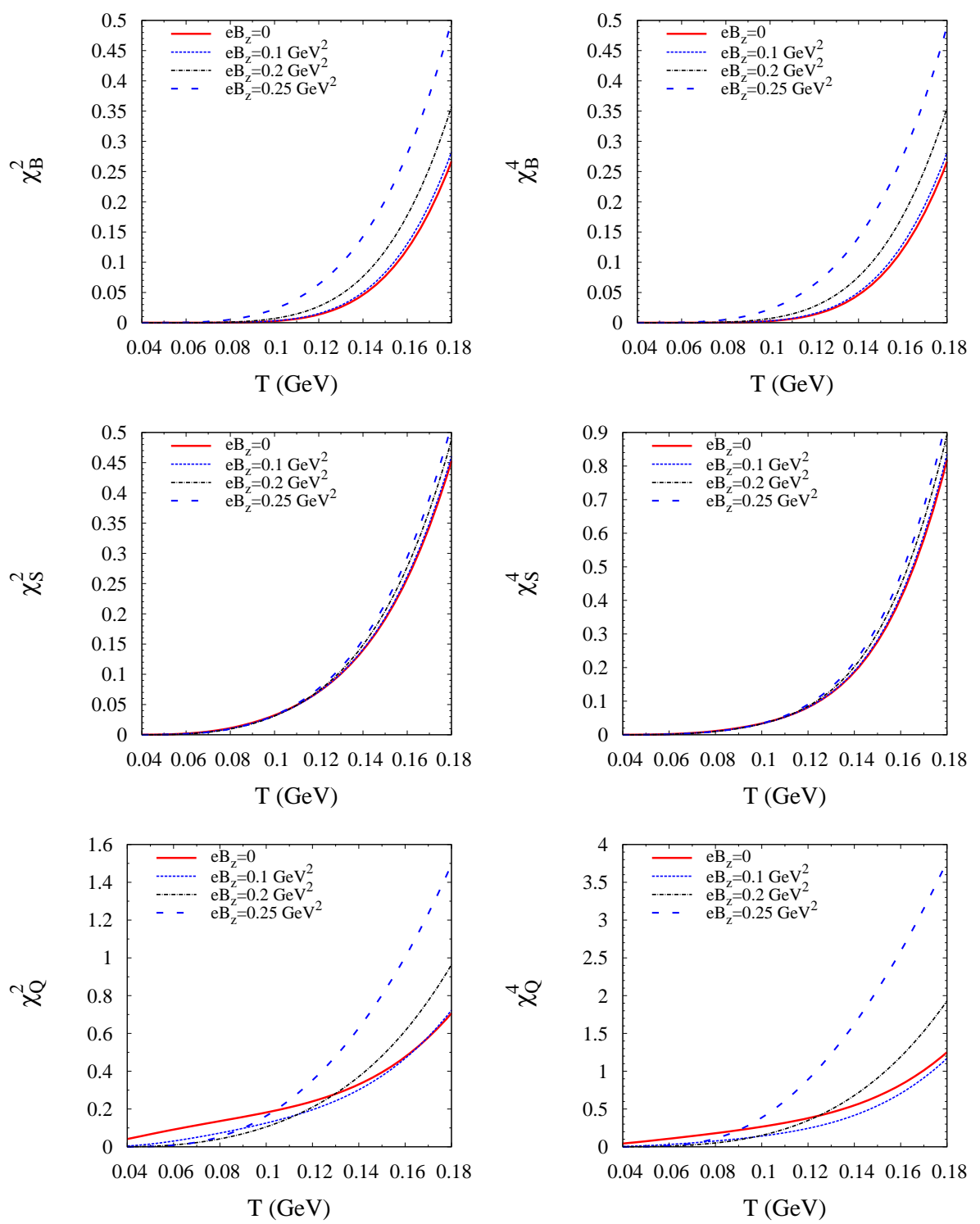

Figure 2: (Color online) Variation of $\chi^{2}$ and $\chi^{4}$ with temperature at $\mu=0$ for baryon number (top row), strangeness (middle row) and electric charge (bottom row).

of magnetic field. As a result susceptibilities of conserved electric charges decrease with increase of magnetic field at low temperature. With increase of temperature $\rho, K^{*}, p, \Delta$ etc., which are spin non-zero particles, appear in the system and these particles cause the susceptibilities to rise at high temperature and high magnetic field.

\section{Acknowledgement}

The work is funded by Council for Scientific and Industrial Research (CSIR), Department of Science and Technology (DST) and Alexander von Humboldt (AvH) foundation. 


\section{References}

[1] S. Chakrabarty and P. K. Sahu, Phys.Rev. D 53 (1996) 4687.

[2] S. Chakrabarty, Phys.Rev. $\mathbf{D 5 4}$ (1996) 1306.

[3] X.-J. Wen, S.-Z. Su, D.-H. Yang and G.-X. Peng, Phys. Rev. D 86 (2012) 034006.

[4] M. Sinha, X. G. Huang and A. Sedrakian, Phys.Rev. D 88v(2013) 025008.

[5] V. Skokov, A. Y. Illarionov and V. Toneev, Int. Jr. Mod. Phys. A 24 (2009) 5925.

[6] T. Schafer and E. V. Shuryak, Rev. Mod. Phys. 70, 3231998.

[7] D. E. Kharzeev, L. D. McLerran, and H. J. Warringa, Nucl. Phys. A 803 (2008) 227.

[8] K. Fukushima, D. E. Kharzeev and H. J. Warringa, Phys. Rev. D 78 (2008) 074033.

[9] P. V. Buividovich, M. N. Chernodub, E. V. Luschevskaya and M I. Polikarpov, Phys. Rev. D 81 (2010) 036007.

[10] B. I. Abelev et al, Phys.Rev.Lett. 103 (2009) 251601.

[11] B. I. Abelev et al, Phys. Rev. C 81 (2010) 054908.

[12] L. Adamczyk et al, Phys. Rev. C 89 (2014) 044908.

[13] L. Adamczyk et al, Phys. Rev. Lett. 113 (2014) 052302.

[14] N. N. Ajitanand, R. A. lacey, A. Taranenko and J. M. Alexander, Phys. Rev. C83 (2011) 011901(R).

[15] I. Selyuzhenkov et. al, Prog.Theor.Phys.Suppl. 193 (2012) 153.

[16] I. A. Shovkovy, Lect. Notes Phys. 871 (2013) 13.

[17] F. Preis, A. Rebhan, A. Schmitt, JHEP 1103 (2011) 033.

[18] F. Bruckmann, G. Endrodi, T. G. Kovacs, JHEP 1304 (2013) 112.

[19] G. S. Bali, F. Bruckmann, G. Endrodi, Z. Fodor, S. D. Katz, S. Krieg, A. Schafer and K. K. Szabo, JHEP 1202 (2012) 044.

[20] M. D’Elia, S. Mukherjee, F. Sanfilippo, Phys. Rev. D 82 051501(R)

[21] A. J. Mizher, M. N. Chernodub and E. S. Fraga, Phys.Rev. D 82 (2010) 105016

[22] V. Skokov, Phys. Rev. D 85 (2012) 034026.

[23] K. Fukushima, M. Ruggieri and R. Gatto, Phys.Rev. D 81 (2010) 114031.

[24] W. Fu, Phys. Rev. D 88 (2013) 014009.

[25] G. Endrődi, JHEP 1304 (2013) 023.

[26] P. Braun-Munzinger, K. Redlich and J. Stachel, in Quark Gluon Plasma 3, World Scientific Publishing (2004).

[27] P. Braun-Munzinger, J. Stachel, J. P. Wessels and N. Xu, Phys. Lett. B 344 (1995) 43.

[28] P. Braun-Munzinger, I. Heppe and J. Stachel, Phys. Lett. B 465 (1999) 15.

[29] P. Braun-Munzinger, D. Magestro, K. Redlich and J. Stachel, Phys. Lett. B 518 (2001) 41.

[30] A. Andronic, P. Braun-Munzinger, J. Stachel and M. Winn, Phys. Lett. B 718 (2012) 80.

[31] A. Bhattacharyya, S. Das, S. K. Ghosh, R. Ray, and S. Samanta, Phys. Rev. C 90 (2014) 034909.

[32] L. D. Landau and E. M. Lifshitz, Quantum Mechanics, Pergamon Press, (1965).

[33] A. Bhattacharyya, S. K. Ghosh, R. Ray, and S. Samanta, arXiv:1504.04533 [hep-ph]. 